\title{
Two novel mutations of pfdhps K540T and I588F, affecting sulphadoxine-pyrimethamine-resistant response in uncomplicated falciparum malaria at Banjar district, South Kalimantan Province, Indonesia
}

\author{
Sukmawati Basuki ${ }^{1,2,3}$, Fitriah $^{3}$, Sugeng Riyanto ${ }^{4}$, Budiono ${ }^{5}$, Yoes P Dachlan ${ }^{2,6}$ and Haruki Uemura $^{7 *}$
}

\begin{abstract}
Background: Mutations in pfdhfr and pfdhps genes have been shown to associate with sulphadoxine-pyrimethamine (SP) resistance of Plasmodium falciparum parasites. However, pfdhfr, pfdhps genotypes and the correlations to SP treatment outcome in Indonesia has not yet been well analysed.

Methods: After obtaining informed consent, 61 uncomplicated falciparum malaria patients were recruited in Banjar district, South Kalimantan Province, Indonesia, from October 2009 to August 2010. They were treated by a single oral dose of SP and its effects on clinical and parasitological status were followed until day 28 after treatment. Occasionally, a thick smear blood film for microscopy observation and blood spot on a filter paper for pfdhfr and pfdhps genotype analysis were collected.

Results: Pfdhfr and pfdhps genotypes from 24 P. falciparum-infected patients consisting of adequate clinical parasitological response (ACPR) $(n=6 ; 25.0 \%)$ and early treatment failure (ETF) $(n=10 ; 41.7 \%)$ or late parasitological failure (LPF) $(n=8 ; 33.3 \%)$ were obtained by sequencing. Two novel mutations of pfdhps gene, K540T and I588F, were determined in ten and five isolates, respectively. These mutations were present in the pfdhfr/pfdhps combined haplotypes of ANRNI/SGTGA $(n=6)$, ANRNL/SGTGA $(n=4)$, and ANRNI/SGEAA(588F) $(n=5)$, (mutation codons are bold typed); these haplotypes were mostly belonging to parasitological failure (ETF or LPF). The parasites acquiring five mutations in pfdhfr/pfdhps haplotypes and four mutations with additional 1588F did not respond adequately to SP treatment.

Conclusion: Many of Plasmodium falciparum infected patients in Banjar district, South Kalimantan, Indonesia did not respond adequately to SP treatment and these low ineffectiveness of SP in this area was associated with two novel mutations of pfdhps, K540T and I588F.
\end{abstract}

Keywords: Plasmodium falciparum, Sulphadoxine-pyrimethamine, pfdhfr, pfdhps

\footnotetext{
* Correspondence: uemura@nagasaki-u.ac.jp

${ }^{7}$ Department of Protozoology, Institute of Tropical Medicine, Nagasaki

University, 1-12-4 Sakamoto, Nagasaki 852-8523, Japan

Full list of author information is available at the end of the article
} 


\section{Background}

Malaria is one of the important public health problem in Indonesia, causing annual parasitic incidence of 1.85\% and malarial outbreaks in several endemic areas leading 11 deaths in 2009 [1,2]. One of the major problems in Indonesia is parasite resistance to anti-malarial drugs. High rates of chloroquine resistance in falciparum and vivax malaria have been reported in Indonesia and sulphadoxine-pyrimethamine (SP) resistance in falciparum malaria was discovered in several endemic areas from 1981 to 1995. In vitro and in vivo SP resistance were found in four provinces: Central Java, East Timor, South Sulawesi, and Papua in 1991 by Tjitra et al. [3] and in vitro resistance was confirmed by using field isolates obtained from 11 malaria-endemic provinces, including East Kalimantan Province [3,4]. In spite of these reports, people in several endemic areas still commonly use SP when they are suffering from malaria because of its effectiveness, fewer side effects, low cost, and the single oral dose treatment. Additionally, SP is provided for chemoprophylaxis [5].

Efficiency of SP for falciparum malaria treatment could be predicted by analysis of Plasmodium falciparum target genes. Pyrimethamine and sulphadoxine inhibit the dihydrofolate reductase (PfDHFR) and dihydropteroate synthase (PfDHPS) activities of the folate biosynthesis pathway in the parasite. However, point mutations in each of the genes, $p f d h f r$ and $p f d h p s$, cause conformational changes in the enzyme structures and result in prevention of adequate drug binding and parasite resistance to SP. The amino acid substitutions at 16, 50, 51, 59, 108 and 164 in PfDHFR are responsible for resistance to pyrimethamine, are predicted stepwise mechanisms of mutations beginning at the codon 108 from serine to asparagine (S108N), and proceeding accumulations of N51I, C59R and I164L mutations confer high-level resistance. Similarly, resistance to sulphadoxine is induced by point mutations of the codons at amino acid positions 436, 437, 540, 581 and 613 in pfdhps gene, which also may progress in certain order, beginning primarily with alanine to glycine change at codon 437 (A437G), followed by K540E and A581G, then other mutations [6-14]. Mutations of $p f d h f r$ and pfdhps have also correlated with SP resistance in vivo by triple mutant of pfdhfr at positions N51I, C59R, S108N and of quintuple mutant of pfdhfr/ pfdhps combination with additional mutant of pfdhps at positions A437G and K540E [9,15-20].

In Indonesia, there are not many reports about genotype analysis of $p f d h f r$ and pfdhps and their correlation with SP treatment outcome. Nagesha et al. reported in 2001 with West Papua parasites obtained in 1996-99 that polymorphisms at C59R and S108N of pfdhfr, and at A437G and K540E of pfdhps were closely associated with resistance [21]. They mentioned no mutations were observed at codons 16, 50, 51 and 164 of the pfdhfr gene and at 436, 581 and 613 of the pfshps gene [21]. Syafruddin et al. expanded the analysis to eight malaria-endemic areas, representing a broad region of the eastern and western Indonesian archipelago and presented additional polymorphisms in pfdhfr gene at the codons of A16V and S108T from several island parasite samples, the more common distributions being among eastern parts [22]. In this report, they mentioned less frequency of pfdhps polymorphisms; most of the parasites presented wild type pfdhps and about $15 \%$ of $437 \mathrm{G}$; less than $5 \%$ of K540E mutations were detected [22]. Recently analysed $p f d h f r$ and $p f d h p s$ genotypes, using a small number of parasite samples from South Kalimantan, by PCR-RFLP method were presented with complete mutations at $\mathrm{S} 108 \mathrm{~N}$ of $p f d h f r$ and A437G of pfdhps, in addition pfdhps K540E substitution from one patient's parasites [23].

In this study, sequence polymorphisms of $p f d h f r$ and pfdhps genes, and these correlations to SP treatment outcome were conducted in 2009-10 in South Kalimantann Province, Indonesia. It will be helpful to estimate SP effectiveness in many situations.

\section{Methods}

\section{Study site and participants}

The study was conducted at Sei Pinang and Aranio subdistricts, Banjar district, South Kalimantan Province, Indonesia (Figure 1) from October 2009 to August 2010, and was approved by the Ethical Committees, Faculty of Medicine, Universitas Airlangga, Surabaya, Indonesia and Institute of Tropical Medicine, Nagasaki University, Japan. Total population of the study site subdistricts was 25,975 in 2008 and the most common occupations were farmers and mining workers. Both subdistricts have high prevalence of malaria. Written informed consent was obtained from each participant, or from caretakers if participants were under 12 years of age, after explanation in the local language of the study procedure.

Symptomatic falciparum malaria patients without complications were recruited by local study teams, which consisted of nurses and microscopists from the regional health centre. Both P. falciparum alone and $P$. falciparum infections mixed with other malaria parasites, diagnosed by Now ${ }^{\circ}$ Malaria ICT diagnosis kit (Binax Inc, Portland, ME, USA), were selected from uncomplicated falciparum malaria patients and treated with a single oral dose of $25 \mathrm{mg} / \mathrm{kg}$ sulphadoxine: $1.25 \mathrm{mg} / \mathrm{kg}$ pyrimethamine tablet (PT Kimia Farma Tbk. DOEN (Daftar Obat Esensial Nasional)), which was contributed by Ministry of Health, Indonesia. Patients took the medicine in front of a member of the team. Patient follow-up was conducted by the teams, by either a team member visiting a patient at home or the patient visiting the health centre, for the purpose of obtaining information about clinical improvement or side 


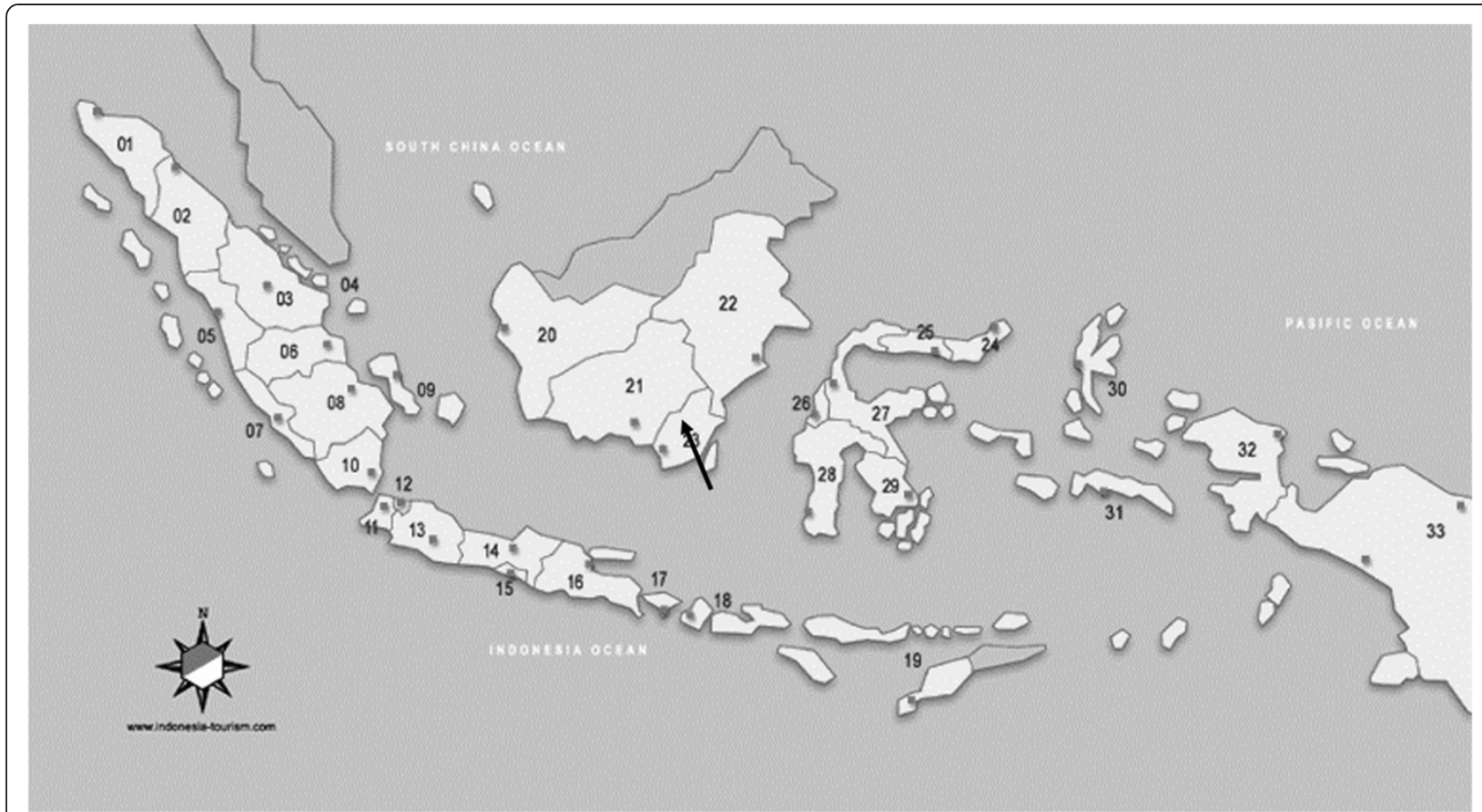

Figure 1 Study site, Banjar district, South Kalimantan Province, Indonesia.

effects of the treatment, for 28 days, as described by WHO [24]. On each occasion, a finger-prick blood sample was taken to prepare a thick blood smear film for microscopical observation and a filter-paper blood spot for DNA extraction (on day 7 and day 21). Patients that failed treatment with SP were treated with a combination of quinine and primaquine on day 28. Treatment outcomes were assessed using the 2009 WHO standard definition for clinical and parasitological responses: adequate clinical parasitological response (ACPR), early treatment failure (ETF), late clinical failure (LCF), late parasitological failure (LPF) [25].

\section{Plasmodium falciparum isolates Microscopy}

Thick smear blood films stained with Giemsa solution (Merck, Germany) were performed for P. falciparum diagnosis. The parasite density was determined by counting asexual parasites in each 200 white blood cells, then converted as the number of asexual parasites per $\mu$ l blood with consideration of an assumed white blood cell density (8 000 per $\mu \mathrm{l}$ ) [26].

\section{Mutation analysis}

Parasite DNA was isolated from the dried blood spots on filter paper (Advantec, Toyo Roshi Kaisha Ltd, Japan) using QIAamp DNA blood mini kit (Qiagen, the Netherlands) as described by Sakihama et al. [27]. Plasmodium falciparum was identified by nested PCR technique using primers of
Snounou et al. and Kimura et al. [28,29]. Pfdhfr and pfdhps genotypes were determined by sequence analysis as previously described by Isozumi et al. [30]. The amplified PCR products were directly used as templates for sequencing with a BigDye Terminator (version 1.1) cycle sequencing kit and a model 3730 genetic analyzer (Applied Biosystems, USA). Alleles at residues 16, 51, 59, 108, and 164 of the pfdhfr, and at residues 436, 437, 540, 581, and 613 of the pfdhps were read carefully, and two independent PCR products were subjected to sequence analysis in the case of new or rare mutations.

\section{Statistical analysis}

Data were entered in Microsoft Excel and exported to SPSS version 17.0 for analysis. The strength of an association was evaluated by calculating odds ratios (OR). Chisquare and Fisher's exact tests were used, where applicable, to assess the relationship between single and multiple mutations to treatment outcomes. Arithmetical means, median and percentage were calculated, where applicable, for subject characteristics and treatment outcomes. Allele proportions were calculated as the number carrying a certain allele divided by the number of samples with positive PCR outcomes.

\section{Results}

Patient characteristics and treatment outcomes

A total of 87 malaria patients were identified at primary health centres. However, 22 malarial patients were 
unwillingness to participate in this study, one participant dropped out from follow-up on day 2 after treatment because of moving from the study site, and three vivax malaria patients were excluded. Thus, a total of 61 falciparum malaria patients treated with SP, who had characteristics as presented in Table 1, were included in this analysis. One malaria patient with mixed infections, which were identified by microscopy and PCR as falciparum and vivax malaria, was involved in the study.

SP treatment resulted in $36.1 \%$ of ACPR and $63.9 \%$ of parasitological failures; $26.2 \%$ of ETF and $37.7 \%$ of LPF. Late clinical failure were not observed in this study (Table 1) and no adverse reactions were identified. Although there were many cases of ETF and LPF, the actually detected parasites were few in number in many cases and the parasite-positive rates were decreasing gradually from 39 microscopically positive cases $(63.9 \%)$ on day 7 to 14 cases $(23.0 \%)$ on day 28 (Table 1 , presented as \% of clearance cases). In these cases, the sexual stage parasites were also disappearing consistently and 43 cases (70.5\%) were negative in both asexual and sexual stage parasites on day 28.

\section{Pfdhfr and pfdhps genotypes}

The $p f d h f r$ and $p f d h p s$ genotypes were obtained by DNA sequencing of PCR products from $P$. falciparum-infected patients on the day before treatment (D0) and those from parasite positives on the day after treatment (D7 and D21). The whole set genotypes of pfdhfr and pfdhps

Table 1 Subject characteristics and treatment outcomes

\begin{tabular}{|c|c|c|}
\hline Characteristics & \multicolumn{2}{|c|}{ No of patients $(n=61)$} \\
\hline Median age in years (range) & \multicolumn{2}{|c|}{$25(14-55)$} \\
\hline Male: Female & \multicolumn{2}{|l|}{ 49: 12} \\
\hline Mean parasite density of D0 (per $\mu$ l) (range) & \multicolumn{2}{|c|}{$2,835(40-38,368)$} \\
\hline Adequate clinical parasitogical response (\%) & \multicolumn{2}{|l|}{$22(36.1)$} \\
\hline Parasitological failure* (\%) & \multicolumn{2}{|l|}{$39(63.9)$} \\
\hline Early treatment failure (ETF) & \multicolumn{2}{|l|}{$16(26.2)$} \\
\hline Late parasitological failure (LPF) & \multicolumn{2}{|l|}{$23(37.7)$} \\
\hline Late clinical failure (\%) & \multicolumn{2}{|l|}{0} \\
\hline Parasite clearance ${ }^{* *}(\%)$ : & A) & B) \\
\hline On D3 & $18(29.5)$ & $15(24.6)$ \\
\hline On D7 & $22(36.1)$ & $20(32.8)$ \\
\hline On D14 & $35(57.4)$ & $32(52.5)$ \\
\hline On D21 & $40(65.6)$ & $38(62.3)$ \\
\hline On D28 & $47(77.0)$ & $43(70.5)$ \\
\hline
\end{tabular}

*Parasitological failure is either early treatment failure or late parasitological failure cases. The actually detected parasites were few in number in many cases and it makes some cases difficult to determine whether ETF or LPF clearly.

**Parasite clearance was determined by microscopy, when A) the cases that an asexual parasite was not detected even gametocytes were observed on the day after treatment; B) the cases that neither asexual nor sexual parasites were detected on the day after treatment. $\mathrm{D} 0=$ on the day of treatment. were assessed from 27 patients of $P$. falciparum infection on D0, from 15 blood samples of D7 and 18 samples of D21. Based on microscopical observation and genotype analysis, correlations between the parasite genotypes and responses to SP treatment were presented on Table 2. The cases of mixed genotype infections (cases A and C), or different genotypes of $p f d h f r$ or $p f d h p s$ on day 0 and the following days (cases B and C), are presented separately (Table 3). In total, pfdhfr and pfdhps genotypes of 31 P. falciparum parasites were obtained for analysis.

The pfdhfr mutations at amino acid positions of Cys59, Ser108 and Ile164 to Arg (90.3\%), Asn (100\%) and Leu (29\%), respectively were detected (Figure 2). All the parasites carried the S108N substitution and no wild type pfdhfr allele ANCSI (at positions 16, 51, 59, 108, and 164, respectively) was obtained in this study. In total, three different haplotype alleles, ANCNI, ANRNI and ANRNL (bold type indicates the mutated alleles) were detected in this area (Tables 2 and 3). This simple accumulation pattern of $p f d h f r$ mutation genotypes supports the stepwise mechanism of resistant gene evolution hypothesis.

In the pfdhps gene, mutations were detected at the positions of Ala437, Lys540, Ala581 and Ile588 to Gly (96.8\%), Glu or Thr (35.5, 35.5\%), Gly (45.2\%), and Phe (22.6\%), respectively. No other substitutions, including at Ser436 and Ala613, were observed (Figure 2). The high proportion of mutation was detected at the position of Ala437 to Gly, which is regarded as the first and the most important change required for sulphadoxine resistance. Two types of mutation were determined at amino acid position 540; AAA (Lys codon) was changed to GAA (Glu) or ACA (Thr) by different types of single nucleotide substitutions. Lysine (K) to glutamic acid (E) substitution at position 540 is reported from many malaria-endemic areas, however, threonine ( $\mathrm{T}$ ) substitution is unique to $P$. falciparum parasites in South Kalimantan. In addition, the other new substitution was identified at amino acid position 588, which

Table 2 Correlation between pfdhfr/pfdhps genotypes and treatment outcomes to sulphadoxine-pyrimethamine

\begin{tabular}{lllll}
\hline pfdhfr*/pfdhps** & $\begin{array}{l}\text { Prevalence } \\
\text { genotypes }\end{array}$ & \multicolumn{3}{c}{ Treatment outcomes } \\
\cline { 3 - 5 } & $(\mathbf{\%})(\mathbf{n}=\mathbf{2 4})$ & ACPR & ETF & LPF \\
\hline ANCNI/SAKAA & $1(4.1)$ & 1 & & \\
ANRNI/SGEAA & $4(16.7)$ & 2 & 1 & 1 \\
ANRNI/SGEAA(588F) & $5(20.8)$ & 1 & 2 & 2 \\
ANRNI/SGTGA & $6(25.0)$ & & 2 & 4 \\
ANRNL/SGKAA & $4(16.7)$ & 2 & 1 & 1 \\
ANRNL/SGTGA & $4(16.7)$ & & 4 &
\end{tabular}

*pfdhfr genotypes are presented at amino acid positions of $16,51,59,108$, and 164 .

**pfdhps genotypes are presented at amino acid positions of $436,437,540$, 581, and 613 (and 588).

The bold type indicates amino acid substitution.

$A C P R=$ adequate clinical parasitological response; $\mathrm{LPF}=$ late parasitological failure, ETF = early treatment failure. 
Table 3 Cases with different genotypes of pfdhfr and pfdhps on the day before drug regimen (DO) and on the days after treatment (D7 and D21)

\begin{tabular}{llll}
\hline & \multicolumn{2}{l}{ pfdhfr*/pfdhps** genotypes } \\
\cline { 2 - 4 } Case & D0 & D7 & D21 \\
\hline A & ANCNI/SGKGA & - & - \\
& ANRNI/SGEAA(588F) & & \\
B & ANCNI/SGKGA & ANRNI/SGTGA & ANRNI/SGTGA \\
C & ANRNL/SGKAA & ANRNI/SGEAA(588F) & ANRNI/SGEAA(588F) \\
& & ANRNI/SGKGA & ANRNI/SGKGA \\
\hline
\end{tabular}

*pfdhfr genotypes are presented at amino acid positions of 16, 51, 59, 108, and 164 .

**pfdhps genotypes are presented at amino acid positions of $436,437,540$, 581 , and 613 (and 588).

The bold type indicates amino acid substitution.

ATT (Ile codon) converted to TTT (Phe codon) by a single point mutation is not previously reported. Altogether including these two novel mutations, six pfdhps haplotypes, SAKAA (at positions 436, 437, 540, 581, and 613, respectively), SGKAA, SGEAA, SGKGA, SGTGA and SGEAA (588F) were determined as a single haplotype infection or multiple haplotype infection cases (Tables 2 and 3). The wild type pfdhps allele was present in one case at this study area. The genotypes containing two new mutations were present as the precise haplotypes separately; K540T exists as SGTGA only and I588F mutation was detected in specific haplotype SGEAA(588F). It suggests these new mutations were introduced independently in recent times.

Pfdhfr, pfdhps genotypes and SP treatment outcomes With a combination of three pfdhfr and six pfdhps alleles, eight different combined $p f d h f r / p f d h p s$ haplotypes were determined. Six combined haplotypes from a single genotype infection patient are presented in Table 2. Two additional combined pfdhfr/pfdhps haplotypes, ANCNI/ SGKGA and ANRNI/SGKGA, were detected in multiple genotype infection cases (Table 3). Each case of SP treatment outcome is summarized and presented with a base of combined haplotypes (Table 2).

Although the actually detected parasites were few in number in many cases and it makes such cases difficult to determine whether ETF or LPF clearly, some correlations between $p f d h f r / p f d h p s$ haplotypes and SP sensitivity were observed. The parasites containing ANCNI/ SAKAA and ANCNI/SGKGA combination haplotypes on day 0 were not detectable on day 7 after treatment and these belonged to ACPR. In the quadruple mutant case of ANRNI/SGEAA, ANRNI/SGEAA(588F) and ANRNL/ SGKAA haplotypes (not included new I588F mutation in counting number of mutations), both ACPR and parasitological failure responses (ETF or LPF) were observed. All cases of parasites presenting ANRNI/SGTGA and ANRNL/SGTGA haplotypes, both of which contain new K540T substitution, on day 0 were not cleared on day 7 and these were included in a group of parasitological failure (ETF or LPF); the former quintuple mutant was ETF or LTF and the latter sextuple case was all ETF. The quintuple or more mutant parasites acquiring more than five mutations in pfdhfr/pfdhps haplotypes did not respond adequately to SP treatment (Table 4). The new I588F mutation was observed only in the parasites of ANRNI/SGEAA(588F) haplotype and comparison of those with or without I588F mutation indicates this amino acid substitution contributes insufficient response of the parasites to SP treatment (Tables 2 and 3).

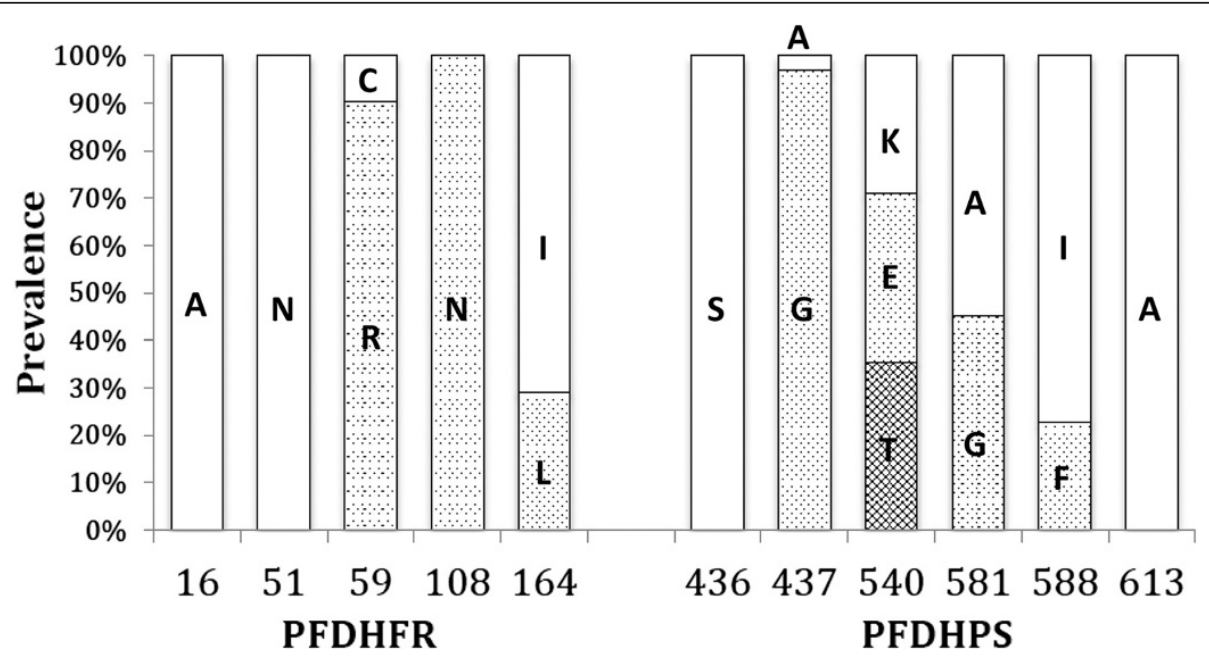

Figure 2 Prevalence of the mutation pfdhfr and pfdhps genotypes. The horizontal axis shows the position or codon of the mutation pfdhfr and pfdhps genes. The white box indicates the wild type and the texture boxes are mutants written by amino acid abbreviations. $A=a$ lanine, $\mathrm{N}$ = asparagine, $\mathrm{C}=$ cysteine, $\mathrm{R}=$ arginine, $\mathrm{I}=$ isoleucine, $\mathrm{L}=$ leucine $\mathrm{S}=$ serine, $\mathrm{G}=$ glycine, $\mathrm{K}=$ lysine, $\mathrm{E}=$ glutamic acid, $\mathrm{T}=$ threonine, $\mathrm{F}=$ phenylalanine. 
Table 4 Assessment association between pfdhfr and pfdhps genotypes and treatment outcomes

\begin{tabular}{llllll}
\hline Genotype & \multicolumn{2}{l}{ Treatment outcomes } & 95\% Cl & $\boldsymbol{P}$ \\
\cline { 2 - 3 } & ACPR & ETF/LPF & & \\
\hline$<$ quintuple mutants & 6 & 8 & $1.112-2.755$ & 0.024 \\
$\geq$ quintuple mutants & 0 & 10 & & \\
Total & 6 & 18 & & \\
\hline
\end{tabular}

Quintuple mutants = the cases containing five mutations of pfdhfr $108 \mathrm{~N}, 59 \mathrm{R}$, $164 \mathrm{~L}$ and pfdhps 437G, 540E or 540T, 581G.

$\mathrm{ACPR}=$ adequate clinical parasitological response;

$\mathrm{ETF} / \mathrm{LPF}=$ parasitological failure.

In this follow-up study of SP treatment, different parasite genotypes were determined at day 0 and day 7,21 in two cases (Table 3, B and C). The parasite numbers decreased at day 3 were increased at day 7 in these two cases (data not shown); it suggested a possibility that the increases in parasite numbers were due to new infections and lower responses of these parasites to SP. Indeed both $B$ and $C$ cases, the quintuple mutant ANRNI/SGTGA in $\mathrm{B}$ and the quadruple mutant ANRNI/SGEAA(588F) in C belong the genotypes that were not respond adequately to SP treatment in Table 2.

\section{Discussion}

In this report, polymorphisms of $p f d h f r$ and $p f d h p s$ genes and these correlations with SP treatment outcomes in South Kalimantan, Indonesia were analyzed. The codons at amino acid positions of 16, 51, 59, 108 and 164 in pfdhfr gene, and 436, 437, 540, 581 and 613 in pfdhps gene were investigated by DNA sequencing of PCR products from $P$. falciparum-infected patient blood. Amino acid substitutions of these polymorphic positions have been shown to be important for SP resistance $[6,8,20,31,32]$ and mutations at several positions, C59R, S108N and I164L in pfdhfr, A437G, K540E or T, A581G, and additionally I588F in pfdhps gene were observed in this research. In these mutations, K540T and I588F of pfdhps are unique in this study area. With these polymorphisms in pfdhfr and pfdhps genes, three different pfdhfr and six pfdhps haplotype alleles were determined, and then a total of eight pfdhfr/pfdhps combined haplotypes were assessed. The comparison of the haplotypes supported the stepwise accumulation of mutations in pfdhfr and pfdhps alleles and that it might be caused by intensive use of SP for malaria treatment at the study sites.

The mutation of $\mathrm{K}$ to $\mathrm{E}$ at position 540 of pfdhps had strong correlation with resistance to sulphadoxine and to SP treatment $[6,10,11,33-35]$. The novel mutation K540T of pfdhps at the same amino acid position was identified in this study. It was shown that $P$. falciparum parasites carrying the K540T mutation of pfdhps allele were all found in Parasitological failure, ETF or LPF
(Table 2), and each isolate carrying K540T was always associated to the mutations of A437G and A581G, forming the triple mutant of $p f d h p s$ allele. This accumulation of mutations causes parasite resistance to SP treatment. It also shows the second novel mutation at the position pfdhps 588, I to $\mathrm{F}$ (I588F). This mutation was always observed in association with mutations A437G and K540E forming double mutant of pfdhps (the I588F mutation is not included in counting number of mutations). Most of the parasites presenting the I588F mutation caused Parasitological failure. These findings indicate that these novel mutations could be a predictor of resistance to SP treatment. The different amino acid substitutions at the same position may affect different levels in sulphadoxine inhibition, such that K540N pfdhps brings a lower level of sulpha drug resistance than the mutation $\mathrm{K} 540 \mathrm{E}$ [34]. An analysis study of how strong the K540T mutation in pfdhps affects the parasite response to sulphadoxine is required.

The K540T mutation is recently reported in Sabah, Malaysian Borneo, by Lau et al. [36]. The same unique mutant K540T and SGTGA genotype of pfdhps were found in this study site in South Kalimantan, Indonesia and in Sabah, Malaysian Borneo; these two regions are located on the same island which suggests that the K540T mutation originates on the island or was introduced, as the parasites expressing it, SGTGA genotype of pfdhps, then spread on the island. In the case of Malaysian Borneo, the parasites carrying $\mathrm{K} 540 \mathrm{E}$, previously predominant point mutation at position 540 , was substituted by K540T and the SGTGA type allele of pfdhps gene became the most prevalent allele in that region. In the case of South Kalimantan, it is noteworthy that I588F, the other new mutation in pfdhps and which may contribute to parasite resistance to SP, exists additionally. The origin and spread of these two novel pfdhps mutations in Indonesia is now under investigation.

In this report, eight $p f d h f r / p f d h p s$ combined haplotypes were observed in South Kalimantan, Indonesia and these correlations to SP treatment outcome were presented; more than 63\% parasites did not respond to SP adequately and correlations between pfdhfr/pfdhps genotypes and parasite response to SP were summarized. The parasites presenting less than three mutations in pfdhfr/pfdhps combined haplotypes responded adequately, and in the cases of four mutations in combined haplotypes (I588F mutation is not included for counting the number of mutations), the parasite responses were not consistent, either ACPR, ETF or LPF (Table 2). The parasites harbouring more than five mutations in $p f d h f r / p f d h p s$ combined haplotypes did not always respond sufficiently as reported previously [20]. Even though a limitation in this study that the drug levels in the blood were not measured, the correlations $p f d h f r / p f d h p s$ haplotypes and SP 
treatment outcome could be applicable in other areas to predict SP efficiency in Indonesia.

According to Nagesha and colleagues [21,22], Fansidar (Roche Pharmaceuticals SP)-resistant parasites from West Papua and some areas in Indonesia presented $p f d h f r / p f d h p s$ genotypes of $59 \mathrm{R}+108 \mathrm{~N} / 437 \mathrm{G}+540 \mathrm{E}$. This quadruple pfdhfr/pfdhps combined haplotype observed in this study was also responding either ACPR, ETF or LPF to SP, and it can be understood that the quadruple pfdhfr/pfdhps haplotype is at the beginning of SP resistance. Nagesha et al. did not detect resistant parasites expressing quintuple combined haplotypes at the time of parasite collection, but it is important to analyse the current situation in West Papua and other islands.

It has been investigated whether either pfdhfr or pfdhps genotype has a principal role in parasite resistance to SP treatment $[11,33,35,37]$. The present study and previous works have demonstrated the genotype of $p f d h f r$ is not complicated and the pfdhps is more polymorphic in Indonesian parasites. In the cases of the Kalimantan sample analysis, most of the parasites (90.3\%) harbouring more than two mutations in pfdhfr gene were associated with different types of $p f d h p s$ alleles, SGEAA, SGEAA(588F) and SGTGA, and which are critically important for parasite resistance to SP treatment. The parasites of K540E could be aware of a situation at the beginning of SP resistance, and with an additional I588F mutation tend to some resistance (Table 2). The other K540T substitution is associated with additional mutation A581G and is recognized as SP-resistant (Table 2). Altogether, the genotype of pfdhps, particularly $\mathrm{K} 540 \mathrm{E}$ or $\mathrm{T}$ and $\mathrm{I} 588 \mathrm{~F}$ are important for understanding the parasite SP responses.

The polymorphisms and genotyping of $P$. falciparum drug-resistant genes are in many cases determined by using specific restriction enzyme digestion. In the case of pfdhps I588F mutation, ATT (Ile codon) to AAA (Phe codon) could be distinguished by Dra I digestion; the mutation AAA (Phe) is digested. Unfortunately, it was not possible to identify pfdhps K540T by a simple restriction enzyme digestion. The detection systems to identify single nucleotide differences, such as using hybridization of fluorescence-labelled oligonucleotide, comparison of melting temperature curves of PCR products, or sequencing is required. However, all the pfdhps K540T mutation were detected in the SGTGA genotype of $p f d h p s$ and A581G mutation by PCR-RFLP methodology [38] could be applied as an indirect indication signal for the mutation.

SP should not be considered for therapeutic use in this region of Indonesia. SP efficiency in this study area was only $36.1 \%$ and remaining $63.9 \%$ presented parasites more than one week after treatment. In either ETF or LPF cases, patients did not present severe symptoms and the parasites in the blood were decreasing gradually. It might be one of the reasons local people kept and used SP for malaria treatment.

\section{Conclusion}

Polymorphisms of $p f d h f r / p f d h p s$ genotypes in Banjar district, South Kalimantan, Indonesia, and these correlations with parasite responses to SP treatment were analyzed in this study. The pfdhfr/pfdhps combined haplotypes were clearly related to the treatment outcomes and the two newly found novel pfdhps mutations, K540T and I588F, were important indicators of SP resistance.

\section{Competing interests}

The authors have declared that they have no competing interests.

\section{Authors' contributions}

SB and HU prepared the protocol, analysed and interpreted data, wrote the paper; SR coordinated field study and data collection in location; F analysed samples at the laboratory; B developed protocol; YPD reviewed the protocol, results, interpretation and the manuscript. All authors read and approved the final manuscript.

\section{Acknowledgements}

We are grateful to all participants in this study and staff members of local health centres in Banjar district for their kind assistance.

This work was supported by the Ministry of Health, and by a research grant (to SB) from Ministry of Education and Culture, Republic of Indonesia, and Grant-in-Aid for Scientific Research (KAKENHI, 19406011 and 22406010 to HU) from the Japan Society for Promotion of Science. This research was accomplished by academic cooporation between Nagasaki University and Universitas Airlangga, and partly supported by Nagasaki University advanced promotion programme. SB obtained the Indonesia Ministry of Education and Culture's Sandwich-like Program Scholarship for scientific publication as a part fulfilment of a PhD.

\section{Author details}

${ }^{1}$ Medical Science, Graduate School, Faculty of Medicine, Universitas Airlangga, Jl Mayjen Prof Dr Moestopo 47, Surabaya 60131, Indonesia. ${ }^{2}$ Department of Medical Parasitology, Faculty of Medicine, Universitas Airlangga, Jl Mayjen Prof Dr Moestopo 47, Surabaya 60131, Indonesia. ${ }^{3}$ Malaria Study Group/Laboratory of Malaria, Institute of Tropical Disease, Universitas Airlangga, Kampus C II Mulyorejo, Surabaya 60115, Indonesia. ${ }^{4}$ Department of Health of Banjar District, J Jend A Yani KM 100, Martapura 70611, South Kalimantan Province, Indonesia. ${ }^{5}$ Department of Public Health and Preventive Medicine, Faculty of Medicine, Universitas Airlangga, J Mayjen Prof Dr Moestopo 47, Surabaya 60131, Indonesia. ${ }^{6}$ Tropical Infectious Diseases Hospital, Kampus C Universitas Airlangga, Kampus C II Mulyorejo, Surabaya 60115, Indonesia. ${ }^{7}$ Department of Protozoology, Institute of Tropical Medicine, Nagasaki University, 1-12-4 Sakamoto, Nagasaki 852-8523, Japan.

Received: 23 December 2013 Accepted: 25 March 2014 Published: 4 April 2014

\section{References}

1. Ministry of Health Indonesia: Epidemiologi malaria di Indonesia. Jakarta: Buletin Jendela Data dan Informasi Kesehatan; 2011.

2. Ministry of Health Indonesia: Profil data kesehatan Indonesia tahun 2011. Jakarta: Kementerian Kesehatan Republik Indonesia; 2012

3. Tjitra E, Marwoto HA, Reny M, Ompusungu S, Tuti S: Penelitian obat anti malaria. Penelitian Kesehatan Indonesia 1991, 19:15-22.

4. Tjitra E, Gunawan S, Laihad F, Sulaksono S, Arjono S, Richie TL, Romzan A: Evaluation of antimalarial drugs in Indonesia 1981-1995. Buletin Penelitian Kesehatan Indonesia 1997, 25:27-58.

5. Laksono RD: Profilaksis di Perbatasan Indonesia-Timor Leste. Cermin Dunia Keddokteran 188 2011, 38:503-507.

6. Cowman AF, Morry MJ, Biggs BA, Cross GA, Foote SJ: Amino acid changes linked to pyrimethamine resistance in the dihydrofolate reductase- 
thymidalate synthase gene of Plasmodium falciparum. Proc Natl Acad SCi U S A 1988, 85:9109-9113.

7. Peterson DS, Walliker D, Wellems TE: Evidence that a point mutation in dihydrofolate reductase-thymidylate synthase confers resistance to pyrimethamine in falciparum malaria. Proc Natl Acad Sci U S A 1988, 85:9114-9118

8. Cowman AF: The molecular basis of resistance to the sulfones, sulfonamides, and dihydrofolate reductase inhibitors. In Malaria: parasite biology, pathogenesis, and protection. 1st edition. Edited by Sherman IW. Washington, D.C.: ASM Press; 1998:317-329.

9. Gregson A, Plowe CV: Mechanism of resistance of malaria parasites to antifolates. Pharmacol Rev 2005, 57:117-145.

10. Wang P, Read M, Sims PF, Hyde JE: Sulfadoxine resistance in the human malaria parasite Plasmodium falciparum is determined by mutations in dihydropteroate synthase and an additional factor associated with folate utilization. Mol Microbiol 1997, 23:979-986.

11. Sibley CH, Hyde JE, Sims PF, Plowe CV, Kublin JG, Mberu EK, Cowman AF, Winstaley PA, Watkins WM, Nzila AM: Pyremethamine-sulfadoxine resistance in Plasmodium falciparum: what next? Trends Parasitol 2001, 17:582-588.

12. Triglia T, Wang P, Sims PF, Hyde JE, Cowman AF: Allelic exchange at the endogenous genomic locus in Plasmodium falciparum proves the role of dihydropteroate synthase in sulfadoxine-resistant malaria. EMBO J 1998, 17:3807-3815.

13. Berglez J, lliades P, Sirawarapon W, Coloe P, Macreadie I: Analysis Escherichia coli of Plasmodium falciparum dihydropteroate synthase (DHPS) alleles implicated in resistance to sulfadoxine. Int J Parasitol 2004, 34:95-100.

14. Vanichtanankul J, Taweechai S, Uttamapinant C, Chitnumsub P, Vilaivan T, Yuthavong Y, Kamchonwongpaisan S: Combined spatial limitation around residues 16 and 108 of Plasmodium falciparum dihydrofolate reductase explains resistance to cycloguanil. Antimicrob Agents Chemother 2012, 56:3928-3935

15. Omar SA, Adagu IS, Warhurst DC: Can pretreatment screening for dhps and dhfr point mutations in Plasmodium falciparum infections be used to predict sufadoxine-pyrimethamine treatment failure? Trans $R$ Soc Trop Med Hyg 2001, 95:315-319.

16. Kublin JG, Dzinjalamala FK, Kamwendo DD, Malkin EM, Cortese JF, Martino LM: Molecular markers for failure of sulfadoxine-pyrimethamine and chlorproguanil-dapsone treatment of Plasmodium falciparum malaria. $J$ Infect Dis 2002, 185:380-388.

17. Mugittu K, Ndejembi M, Malisa A, Lemnge M, Premji Z, Mwita A, Nkya W, Kataraihya J, Abdulla S, Beck H-P, Mshinda H: Therapeutic efficacy of sulfadoxine-pyrimethamine and prevalence of resistance markers in Tanzania prior to revision of malaria treatment policy: Plasmodium falciparum dihydrofolate reductase and dihydropteroate synthase mutations in monitoring in vivo resistance. Am J Trop Med Hyg 2004, 72:696-702

18. Staedke SG, Sendagire H, Lamola S, Kamya MR, Dorsey G, Rosenthal PJ: Relationship between age, molecular markers, and response to sulfadoxine-pyrimethamine treatment in Kampala, Uganda. Trop Med Int Health 2004, 9:624-629.

19. Plowe CV: The evolution of drug-resistant malaria. Trans R Soc Trop Med Hyg 2009, 103:S11-S14.

20. Picot S, Olliaro P, de Mobrison F, Bienvenu L, Price RN, Ringwald P: A systematic review and meta-analysis of evidence for correlation between molecular markers of parasite resistance and treatment outcome in falciparum malaria. Malar J 2009, 8:1-15.

21. Nagesha HS, Syafrudin D, Casey GJ, Augustina I, Susanti, Fryauff DJ, Reeder JC, Cowman AF: Mutation in the pfmdr1, dhfr and dhps genes of Plasmodium falciparum are associated with in vivo drug resistance in West Papua, Indonesia. Trans R Soc Trop Med Hyg 2001, 95:43-49.

22. Syafruddin D, Asih PBS, Casey GJ, Maguire J, Baird K, Nagesha HS, Cowman $\mathrm{AF}$, Reeder JC: Molecular epidemiology of Plasmodium falciparum resistance to antimalarial drugs in Indonesia. Am J Trop Med Hyg 2005, 72:174-181.

23. Fitriah, Sulistyawati S, Riyanto S, Budiono, Basuki S, Dachlan YP, Uemura H: Polymorphism of Plasmodium falciparum dihydrofolate reductase and dihydropteroate synthase genes among pregnant women with falciparum malaria in Banjar district, South Kalimantan province, Indonesia. J Trop Life Sci 2012, 2:92-98.
24. WHO: Assessment of theraupetic efficacy of antimalarial drugs for uncomplicated falciparum malaria in areas with intense transmission. Geneva, Switzerland: World Health Organization; 1996.

25. WHO: Methods for surveillance of antimalarial drug efficacy. Geneva, Switzerland: World Health Organization; 2009 [ISBN 9789241597531].

26. Indonesia Directorate General of Diseases Control and Promotion Environment: Pedoman penatalaksanaan kasus malaria di Indonesia. Jakarta: Departemen Kesehatan Rl; 2008 [614.532 Ind m].

27. Sakihama N, Mitamura T, Kaneko A, Horii T, Tanabe K: Long PCR amplification of Plasmodium falciparum DNA extracted from filter paper blots. Exp Parasitol 2001, 97:50-54.

28. Snounou G, Viriyakosol S, Zhu XP: High sensitivity of detection of human malaria parasites by the use of nested polymerase chain reaction. Mol Biochem Parasitol 1993, 61:315-320.

29. Kimura M, Kaneko O, Liu Q, Zhou M, Kawamoto F, Wataya Y, Otani S, Yamaguchi $Y$, Tanabe K: Identification of the four species of human malaria parasites by nested PCR that targets variant sequences in the small subunit rRNA gene. Parasitol Int 1997, 46:91-95.

30. Isozumi R, Uemura H, Dao LD, Van Hanh T, Giang ND, Vien HV, Phuc BQ, Tuan NV, Nakazawa S: Longitudinal survey of Plasmodium falciparum infection in Vietnam: characteristics of antimalarial resistance and their associated factors. J Clin Microbiol 2010, 48:70-77.

31. Brooks DR, Wang P, Read M, Watkins WM, Sims PF, Hyde JE: Sequence variation of the hydroxymethyldihydropterin pyrophosphokinase: dihydropteroate synthase gene in lines of the human malaria parasite, Plasmodium falciparum, with differing resistance to sulfadoxine. Eur J Biochem 1994, 224:397-405.

32. Venkatesan M, Alifrangis M, Cooper C, Plowe CV: Monitoring antifolate resistance in intermittent preventive therapy for malaria. Trends Parasitol 2013, 29:497-504.

33. Dorsey G, Dokomajilar C, Kiggundu M, Staedke SG, Kamya MR, Rosenthal PJ: Principal role of dihydropteroate synthase mutations in mediating resistance to sulfadoxine-pyrimethamine in single-drug and combination therapy of complicated malaria di Uganda. Am J Trop Med 2004, 71:758-763.

34. Lumb V, Sharma YD: Novel K540N mutation of Plasmodium falciparum dihydropteroate synthetase confers a lower level of sulfa drug resistance than does a K540E mutation. Antimicrob Agents Chemother 2011, $55: 2481-2482$.

35. Foote SJ, Cowman AF: The mode action and the mechanism of resistance to antimalarial drugs. Acta Trop 1994, 56:157-171.

36. Lau TY, Sylvi M, William T: Mutational analysis Plasmodium falciparum dihydrofolate reductase and dihydropteroate synthase genes in the interior division of Sabah, Malaysia. Malar J 2013, 12:445. http://www. malariajournal.com/content/12/1/445.

37. Abdul-Ghani R, Farag HF, Allam AF: Sulfadoxine-Pyrimethamine resistance in Plasmodium falciparum: a zoom image in the molecular level within a geographic context. Acta Trop 2013, 125:163-190.

38. Duraisingh MT, Curtis J, Warhurst DC: Plasmodium falciparum: detection of polymorphisms in the dihydrofolate reductase and dihydropteroate synthetase genes by PCR and restriction digestion. Exp Parasitol 1998, 89:1-8.

doi:10.1186/1475-2875-13-135

Cite this article as: Basuki et al: Two novel mutations of pfdhps K540T and $1588 \mathrm{~F}$, affecting sulphadoxine-pyrimethamine-resistant response in uncomplicated falciparum malaria at Banjar district, South Kalimantan Province, Indonesia. Malaria Journal 2014 13:135. 